Service social

\title{
Le bénévolat : un langage du cœur et de raison
}

\section{Suzie Robichaud}

Volume 43, numéro 2, 1994

Enfants, parents, intervenants

URI : https://id.erudit.org/iderudit/706660ar

DOI : https://doi.org/10.7202/706660ar

Aller au sommaire du numéro

Éditeur(s)

École de service social de l'Université Laval

ISSN

1708-1734 (numérique)

Découvrir la revue

Citer cet article

Robichaud, S. (1994). Le bénévolat : un langage du cœur et de raison. Service social, 43(2), 129-146. https://doi.org/10.7202/706660ar

\section{Résumé de l'article}

Le réaménagement de l'État dans l'espace social permet de constater qu'il modifie le paysage des groupes bénévoles et les entraîne dans une logique d'institutionnalisation. Ces groupes adoptent alors une forme de système plus hiérarchique appelé un quasi-appareil (embauche de permanents, spécialisation des acteurs, tendance à l'officialisation, etc.). Mais les changements structurels qui surviennent au sein des groupes modifient moins l'existence du bénévolat que les cadres de son accomplissement. Malgré la diminution de l'effectif bénévole et le déclin de la séduction pour une spontanéité qui se modifie en s'institutionnalisant, la lecture des chiffres invite ici à soutenir le contraire, puisque les personnes interrogées sont plutôt enclines à affirmer qu'elles veulent persister dans la pratique bénévole en dépit de la transformation des groupes. 


\section{Le bénévolat: un langage de cour et de raison}

Suzie ROBICHAUD

Professeure

Département des sciences humaines Université du Québec à Chicoutimi

Nous donnons toujours aisément ce que I'on n'exige pas de nous et ce qu'on ne nous oblige nullement à donner.

Ivan Gontcharov

L'évolution des dernières années montre avec éloquence que le bénévolat subit actuellement des transformations accélérées (FerrandBechmann, 1992; Larochelle et Robichaud, 1991 ; Lamoureux, 1991 ; Laforest et Redjeb, 1989, etc.). La seule volonté ne peut se suffire à elle-même, se déployer sans une préoccupation du rendement social. La vitalité se conjugue ici à l'adaptabilité, le désir d'aider coexiste avec celui d'orienter l'aide, de la rendre plus performante. Ainsi, au Québec, les groupes bénévoles ne peuvent plus demeurer dans un équilibre permanent, sans ruptures ni mutations, dès lors qu'ils doivent affronter 
les contraintes qu'impose le réaménagement de leurs rapports avec I'État. En esquissant ici les inclinaisons qui se tracent dans un mouvement, les groupes semblent entraînés dans une logique susceptible de les confiner à l'institutionnalisation. Leur mission s'accompagne alors de devoirs qui encadrent les actions (critères à respecter pour I'obtention des subventions, bénéficiaires visés en fonction des priorités ministérielles, etc.). Dans ce contexte, il serait intéressant de poursuivre I'interrogation sous un nouvel angle en se demandant comment les nouveaux cadres de gestion de l'action bénévole incitent celle qui intervient à revoir la perception de son geste, sinon à redéfinir les modes de transcription sociale de sa bene volens.

II nous semble utile de préciser, au point de départ, que la réflexion dégagée dans ces pages $\mathrm{n}^{\prime}$ est pas simplement un questionnement sur le phénomène observé, mais se fonde sur une longue enquête réalisée récemment ${ }^{1}$. L'étude, conduite dans une région du Québec - le Saguenay -, tente d'examiner les groupes bénévoles à vocation sociale. La stratégie de recherche utilisée repose sur une méthodologie de type qualitative et son corpus documentaire est issu de l'interrogation d'une trentaine de personnes (dont seize dirigeantes et treize bénévoles) oeuvrant dans le secteur du soutien à domicile.

Rappelons également pour mieux camper le contexte de ce propos que la recherche comporte trois grandes propositions d'ensemble: a) l'évolution des rapports entre l'État et les groupes bénévoles entraîne I'institutionnalisation de ces derniers; b) I'institutionnalisation tend à transformer les groupes bénévoles en quasi-appareils, ce qui les oblige à consacrer plus de ressources à leur fonctionnement interne et moins aux pratiques bénévoles; c) cette transformation suscite une certaine désaffection des bénévoles, attribuable à la lourdeur des contraintes organisationnelles.

Le cadre du présent article se limite à la vérification de la troisième proposition. Il s'agit donc, dans un premier temps, d'étudier la perception des bénévoles relativement aux changements qui surviennent à l'intérieur de leur groupe et dans lesquels s'engage, en ce tournant siècle, le bénévolat. Celui-ci peut être entendu comme une relation d'aide entre au moins deux personnes dans laquelle l'une apporte à l'autre une aide sans y être obligée et sans attendre de contrepartie de la part de quiconque. Par la suite, il y a lieu de faire émerger la vision des bénévoles, afin de comprendre comment elles conçoivent leur pratique et se représentent leur participation dans un

1. Suzie Robichaud, L'État et les solidarités bénévoles : les enjeux politiques de la gratuité (Thèse de doctorat, Université Laval, 1994). 
groupe. Enfin, I'article tente de répondre à la question suivante: malgré les règles de fonctionnement imposées les bénévoles ${ }^{2}$ désirent-elles poursuivre leur engagement?

\section{LE SOCIAL AU QUOTIDIEN}

Les bénévoles sont conscientes des changements qui surviennent à I'intérieur de leur groupe. Elles s'entendent pour dire que les problèmes sociaux sont de plus en plus complexes et que la population a besoin d'un plus grand nombre de services. L'ampleur de la tâche et les ressources disponibles varient en raison inverse l'une de l'autre. Cette situation les laisse perplexes. Par ailleurs, ces bénévoles jugent plus « organisé » qu'auparavant le bénévolat qu'elles pratiquent actuellement. L'augmentation du nombre de permanents, la diminution du nombre de bénévoles, l'accroissement des demandes de services, la professionnalisation des interventions sont les observations qui rendent compte de ce changement. Mais, de toute évidence, les impacts que soulève cette évolution ne font pas, de leur part, l'objet d'une analyse toujours approfondie. II suffit de discuter avec les bénévoles pour voir qu'au-delà de ces modifications structurelles, c'est l'aide à apporter qui domine la réalité quotidienne. Il y a donc lieu de porter une attention toute particulière à la façon dont les bénévoles perçoivent et vivent les transformations qui surviennent dans leur groupe.

Les opinions se divisent sur la valeur du bénévolat contemporain. Il y a celles (sept sur treize) qui ont la nostalgie d'antan, d'une époque où les gens s'entraidaient davantage et de façon plus spontanée comme à l'occasion des grandes corvées. Les autres considèrent plutôt positivement un bénévolat plus encadré, plus structuré. «Nous possédons plus d'outils pour effectuer notre travail et nous jouissons d'une plus grande visibilité dans le milieu. "Toutefois, toutes soulèvent des inquiétudes lorsque vient le temps d'aborder la question de la relève bénévole. Néanmoins, la réflexion sur le bénévolat d’hier et d'aujour$d^{\prime}$ hui conduit à une vision prospective. Et les bénévoles se projettent dans I'avenir. Voici la réflexion de l'une d'entre elles:

Le danger, c'est de tuer le bénévolat. Les bénévoles vont venir à bout de souffle si l'on se fie trop à elles. Quand on les fait fuir, il devient difficile, voire presque impossible de les

2. Puisque la majorité des personnes bénévoles interrogées sont des femmes (onze sur treize) et qu'elles représentent plus de $84 \%$ de l'effectif bénévole de l'ensemble de la population à l'étude, la forme féminine est utilisée à titre épicène. 
réintégrer. Alors, une certaine prudence s'impose si l'on ne veut pas les brûler. Qui a envie d'ailleurs de consacrer des heures innombrables à accomplir un travail qui n'en finit plus de finir?

$C^{\prime}$ est à travers de tels propos que peut s'exprimer un état de trouble lié à l'incertitude que I'on appréhende. Les femmes sont conscientes que le bénévolat ne peut répondre à toutes les demandes provenant des milieux communautaire et institutionnel. Les bénévoles peuvent apporter une aide à autrui, certes, mais peuvent-elles servir d'instrument de réalisation des politiques sociales? Au reste, de quelque manière que ce rôle leur soit conféré, il importe d'en fixer les limites que sa signification même suggère. Car la bénévole n'a aucune obligation contractuelle envers les groupes auxquels elle donne de son temps. Tout en devant respecter certaines modalités d'intervention, elle peut mettre fin à son engagement au moment qu'elle juge opportun. Le bénévolat est donc un geste volontaire, mais aussi temporaire, et il serait illusoire d'utiliser cette pratique sociale pour répondre d'une manière continue à toutes les nouvelles catégories de population en difficulté que la crise sécrète. Des interventions ponctuelles et limitées semblent davantage s'harmoniser avec leur mission. «Nous faisons notre possible, nous mentionnent la plupart des femmes (onze sur treize), mais notre aide ne produit pas toujours les résultats escomptés. »

Un autre fait attire I'attention des bénévoles: I'augmentation des subventions accordées par l'État. Les femmes semblent en accord avec cette hausse. En effet, elles reconnaissent qu'il serait impossible pour leur groupe de répondre aux besoins du milieu sans une aide financière gouvernementale. Qui plus est, elles sont d'avis que le bénévolat rend possible la distribution de services à un coût moindre que ne le permettent les institutions; de plus, celles-ci disposent bien souvent de ressources insuffisantes pour répondre à toutes les demandes qui leur sont adressées. Dans un contexte de déficit budgétaire, ce facteur d'économie n'est pas négligeable, selon elles. Mais c'est trop dire sans doute qu'elles ignorent les enjeux que soulève le financement étatique. C'est une vision plus à court terme qui les incite à regarder les effets positifs d'un financement par l'État. Ce que les bénévoles soulignent cependant avec force, c'est bien la nécessité de l'entraide dans une société où la croissance des dépenses sociales se conjugue avec une crise économique. Fin du rêve, agonie de l'idéal, enlisement de l'aventure pour entrer dans l'ère des incertitudes, du citoyen-providence, afin que celui-ci occupe une plus grande place dans I'organisation sociale. "Mais le danger, c'est de rendre la gratuité rentable et de lui enlever son caractère de liberté », exprime l'une d'elles. 
L'évolution des groupes bénévoles s'observe donc par une série de transformations progressives et de changements successifs que les bénévoles, à cette phase du parcours, expriment souvent en termes de contraintes et de difficultés à surmonter. Elles racontent en toute simplicité les choses tristes, ou saugrenues, ou gaies qui bouleversent leur pratique.

\section{PROFESSION: BÉNÉVOLE}

\section{Les bénévoles et leurs contraintes}

\section{Formalités}

Processus de sélection

Cours de préparation

à l'action bénévole

Sessions de formation

Ententes sur la durée de l'engagement

\section{Pratiques}

Professionnalisation des interventions et division du travail

Règles de conduite

Production de rapports

Frais rattachés

à l'exercice bénévole

D'entrée de jeu, il est utile de mentionner que la majorité des bénévoles interrogées (douze sur treize) exercent leur action à l'intérieur d'un groupe depuis au moins trois ans et la totalité pratiquent un bénévolat de service. Elles apportent une aide physique et psychosociale à des personnes dans le besoin dans le but de les aider à accomplir leurs activités de la vie quotidienne, de faciliter leurs déplacements et d'améliorer leur qualité de vie. Certaines vont jusqu'à refuser d'occu-per un poste au conseil d'administration, considérant cette fonction comme trop éloignée de leur préoccupation immédiate qui consiste à fournir un appui concret à une personne dans le besoin. La rencontre d'éléments, de sentiments contraires qui animent certains membres exerçant un bénévolat de gestion, c'est-à-dire un travail de participation au fonctionnement du groupe, semble éloigner des femmes de ce type d'engagement. "Je suis amie avec tout le monde et je souhaite le demeurer", exprime une bénévole. De plus, pour plusieurs d'entre elles (dix sur treize), ce sont aux permanentes que reviennent les tâches administratives, comme celle de planifier les activités ou de déterminer les priorités d'action. "Les dirigeantes possèdent une plus grande connaissance des dossiers à l'étude et leur 
travail quotidien les prépare davantage à assumer ce rôle », énonce une bénévole. "Au moment de la mise sur pied du groupe, il a fallu, bien sûr, participer davantage à certaines tâches administratives, puisqu'il n'y avait pas de structure permanente », ajoute une autre.

Le moins que l'on puisse dire, c'est que le bénévolat pratiqué à I'intérieur des groupes est, dans une certaine mesure, moins spontané que celui d'il y a quelques années. À preuve, les tâches qu'exécutent les treize bénévoles sont formalisées, c'est-à-dire qu'elles doivent s'effectuer à l'intérieur de normes établies. Presque la moitié des bénévoles (six sur treize) ont été soumises à un processus de sélection, c'està-dire qu'elles ont au moins dû participer à une entrevue à l'intérieur de laquelle leurs motivations, leurs aptitudes et leur disponibilité étaient examinées. De plus, neuf femmes ont suivi des cours de préparation à l'action bénévole: "Aujourd'hui, je sais comment ne pas prendre le problème des autres sur mes épaules», raconte une bénévole. Cependant, deux femmes seulement ont signé une entente sur la durée de leur engagement. Elles ont accepté d'intervenir dans ce groupe pour une période $d^{\prime} u n$ an et $d^{\prime} y$ effectuer des actions pendant quatre heures par semaine. Toutefois, la majorité des bénévoles (onze sur treize) s'accordent pour dire que leur pratique s'est transformée au cours des dernières années. Les modifications touchent, en l'occurrence, la spécialisation de leur travail et les règles qui l'encadrent.

La professionnalisation s'observe, dans un premier temps, par I'importance accordée à la formation et au perfectionnement. Toutes les bénévoles interrogées se sont inscrites à des sessions de formation depuis leur entrée dans le groupe, afin d'acquérir des connaissances en relation d'aide ou sur des aspects touchant diverses problématiques sociales. Certaines bénéficient de supervisions régulières. L'expression des sentiments sous-jacents à leur pratique sociale que permet cette révision du travail semble, de toute évidence, accroître la motivation des bénévoles qui en bénéficient. Cependant, les femmes affirment qu'actuellement le genre d'activités qu'elles accomplissent favorise une division du travail et conduit à l'individualisme, ce qu'elles ne souhaitent nullement. En effet, les actions se divisent en aide morale (visites amicales, soutien psychosocial) ou en services (organisations de fêtes et de loisirs, accompagnement, transport, travaux de réparation, aide aux personnes à domicile, travaux ménagers, distribution de repas). Cette répartition des tâches conduit certaines bénévoles à réaliser leurs activités dans une approche plutôt holistique, alors que d'autres abordent I'action en utilisant une méthode axée davantage sur un aspect de la personne.

Un regard sur la façon dont se fait le travail permet d'éclairer le sens du propos. La bénévole qui prépare les repas n’est pas celle qui 
va les offrir; la bénévole qui exécute quelques travaux ménagers chez des gens $n^{\prime}$ est pas celle qui fait de l'accompagnement auprès de ces mêmes personnes. Une personne âgée en perte d'autonomie peut donc bénéficier des services de plus de trois bénévoles au cours d'une même semaine. On peut effectivement se poser la question suivante, selon certaines femmes: "Comment diminuer le nombre de bénévoles qui fréquentent un même milieu, afin que les gens puissent jouir d'une certaine stabilité dans l'aide qu'ils reçoivent? » Ce faisant, est-il possible de comprendre les conséquences dans le découpage des activités? Disons que la question demeure ouverte. Du moins faut-il postuler que la division du travail bénévole est un des écueils qui guettent cette pratique sociale, dans la présente décennie. Les femmes constatent, de plus, que les activités qu'elles mènent se déroulent dans un encadrement plus serré.

En effet, certaines formules sont imposées comme ligne directrice de conduite: des règles d'éthique (la confidentialité, la discrétion), les données concernant le cheminement des bénéficiaires. "Nous sommes en quelque sorte les chiens de garde du travail effectué ", énonce une bénévole. De plus, les exigences administratives s'élargissent (fichiers, feuilles de route, statistiques, etc.). Cette étude méthodique des faits laisse perplexes plusieurs d'entre elles (dix sur treize): "Le bénévolat, c'est synonyme de gratuité. Nous ne voulons pas compter. Il devient difficile de calculer le temps que nous consacrons aux personnes dans le besoin. » $\mathrm{D}^{\prime}$ ailleurs, il y a de ces phénomènes qui résistent toujours à une simple sommation quantitative. L'analyse comptable risque de devenir un buttoir insurmontable lorsque I'action humaine engage des expressions d'émotivité, de passion, de générosité, d'épanchement vers autrui. Ainsi, compter peut devenir la meilleure manière de ne pas pouvoir apprécier. La réduction quantitative ne vaut que dans I'ordre des moyens, jamais dans celui des fins. Aider autrui, quelle que soit la forme que prend cette propension, ressort du domaine des aspirations dont les modes d'instrumentation peuvent, certes, être comptabilisés; ce n'est guère le cas pour l'enchantement social qui l'inspire. Qu'à cela ne tienne, elles en font parfois à leur tête et, à I'occasion, ne prennent pas en considération les devoirs qui leur incombent. En somme, elles font comme si...! Comme si rien n'avait changé depuis les dernières années, comme si aucune exigence ne leur était dictée.

Cependant, les demandes insistantes et les pressions qui tendent à contraindre l'action bénévole finissent par atteindre les femmes et certaines arrivent mal à accorder ces nouvelles conditions de pratique avec la liberté dont elles veulent disposer favorablement. Chercher à tout concilier et à admettre les incompatibles peut opposer des forces 
vives qui ne visent qu'un seul objectif: assister autrui. Ce qu'il est essentiel de faire ressortir ici, c'est que les multiples obligations imposées aux bénévoles exercent une pression sur leur pratique, soulèvent des interrogations et des craintes. Mais au-delà de ces remarques, il y a lieu de se demander comment se vit et se présente l'autorité dans le groupe qui les encadre.

Selon les bénévoles, les zones d'influence se partagent entre la directrice et les membres du conseil d'administration. Que le pouvoir provienne de l'une ou de l'autre ne fait pas problème. Ce qui nuit au fonctionnement, ce sont les conflits vécus entre la permanence et les membres du conseil d'administration. Les bénévoles notent également que la structure actuelle a une incidence sur le temps qui leur est consacré. En effet, la moins grande disponibilité des dirigeantes produit, chez les bénévoles, un sentiment d'isolement. Presque la moitié d'entre elles (six sur treize) avouent se sentir moins appuyées que dans le passé par celles-ci. Ce n'est pas un blâme qui leur est adressé, puisqu'elles imputent ce changement à l'accroissement des tâches administratives (recherche de sources de subvention, participation aux tables de concertation, gestion du personnel, etc.). Néanmoins, il appert que I'appui et le soutien que les dirigeantes manifestent à l'égard des femmes contribuent, dans une certaine mesure, à maintenir leur intérêt et leur enthousiasme. Les rencontres qui favorisaient les échanges entre les bénévoles sont aussi de moins en moins fréquentes. Cette diminution les laisse songeuses. "Il est intéressant d'échanger avec d'autres femmes des inquiétudes, de discuter des obstacles que l'on rencontre et des résistances que l'on observe dans l'exercice de notre travail », livre une bénévole.

Le sentiment d'appartenance est, pour les femmes, un facteur qui détermine une attitude positive envers la tâche à accomplir et accroît leur motivation, tout comme l'attachement aux membres du groupe favorise la solidarité. Les convictions et les attitudes des bénévoles produisent donc un ensemble de pratiques conçues comme un moyen de stimuler leur générosité, de prendre une part active dans l'établissement d'une société plus juste et plus humaine. C'est dans cette perspective que naît un sentiment d'appartenance à la communauté. Cette sensibilité à la collectivité mérite d'être soulignée, puisqu'elle révèle l'ampleur de la portée sociale du bénévolat.

Toutefois, les activités bénévoles s'exercent à l'intérieur de règles et d'exigences administratives qui ont pour effet simultané de rendre plus difficile l'accès à la pratique du bénévolat. En effet, douze des treize groupes rencontrés ont connu au cours des trois dernières années précédant l'enquête une diminution de vingt pour cent de leur 
effectif ${ }^{3}$. Évolution surprenante, puisque plusieurs études révèlent des signes évidents de la montée spectaculaire du bénévolat (augmentation de l'effectif bénévole et du nombre d'heures consacrées à leur pratique, accroissement des subventions gouvernementales aux organismes bénévoles, multiplication des centres d'action bénévole, etc. $)^{4}$. Bien sûr, le recrutement semble s'effectuer de façon régulière; il n'en demeure pas moins que cette opération conduit à un certain désenchantement. En l'occurrence, onze des treize bénévoles estiment cette action difficile ou très difficile. Cependant, plusieurs d'entre elles (onze sur treize) déclarent qu'il est plus facile de mobiliser des bénévoles pour des activités ponctuelles. Un exemple l'atteste suffisamment: lorsqu'une cause exige le service d'un jour (financement, voyage, journée sportive, etc.), les bénévoles arrivent en grand nombre. Toutefois, les raisons qui militent en faveur d'un certain désintéressement à l'égard du bénévolat touchent les personnes elles-mêmes ou les exigences liées à cette pratique.

La générosité des personnes n'est certes pas mise en cause, mais le contexte social et économique inquiète, car il semble restreindre les possibilités d'engagement social. Selon les bénévoles, trois raisons principales expliquent la difficulté d'inscrire dans le groupe de nouvelles ressources humaines: a) l'augmentation du nombre des demandes; b) les sollicitations trop nombreuses envers les gens qui s'engagent; c) la résistance des jeunes et des aînés; ceux-là justifient leur refus à l'action par leur situation de non-emploi, ceux-ci évoquent une vie déjà bien remplie. "On est en droit de se demander qui seront les bénévoles de demain", estiment certaines femmes (neuf sur treize).

II serait alarmiste de prétendre que les bénévoles sont une espèce en voie de disparition comme quelques-unes le pensent; mais dans une société qui devra s'assumer davantage, elles revendiquent des façons de réinventer la générosité afin que les solidarités immédiates continuent de resurgir. Une aidante à la retraite exprime: "Comme le disait ma mère autrefois, il faut réinstaller le bénévolat. » Faut-il voir dans cette dernière réflexion un questionnement qui traverse le temps et les âges, puisqu'en la décennie 90 l'interrogation subsiste? Au-delà des formalités exposées plus haut, d'autres contraintes semblent gêner

3. La dernière enquête de Statistique Canada (1987) révèle que la participation des bénévoles est moins grande dans les organismes voués à la santé et aux services sociaux. De plus, Blanchet (1990) montre que c'est le bénévolat de services directs qui entraîne les plus nombreuses sources de désillusion pour les bénévoles.

4. Voir à ce propos les recherches de Carpentier et Vaillancourt, 1990 ; Laforest et Redjeb, 1989 ; Duchesne, 1989 ; Payette et Vaillancourt, 1983. 
le bénévolat en cette fin de siècle : les exigences du soutien à domicile et la mesure Expérience de travail, les exigences des bénéficiaires, la vente de services, l'impact de la pratique bénévole sur l'emploi.

\section{Le bénévolat et ses nouvelles contraintes}

- Les exigences du soutien à domicile et la mesure Expérience de travail

- Les exigences des bénéficiaires

- La vente de services

- L'impact de la pratique bénévole sur l'emploi

Les exigences du soutien à domicile et la mesure Expérience de travail

Le maintien des personnes âgées et en perte d'autonomie dans leur milieu naturel ne peut se faire sans l'allégement des contingences de la vie quotidienne qui constituent, pour plusieurs d'entre elles, une charge trop lourde à supporter. L'ensemble des soins matériels, des travaux d'entretien et de propreté requiert une aide extérieure et entraîne une relation nécessaire, inéluctable, avec ceux qui offrent le service. Qui plus est, les demandes de services comportent des différences qualitatives importantes. Ainsi, I'accompagnement, le déneigement, I'entretien lourd ou léger, I'alimentation, le maintien d'un logement autonome constituent les tâches particulières que l'on doit accomplir auprès des bénéficiaires. Peut-on compter sur une maind'œuvre bénévole pour effectuer, par exemple, l'ensemble des tâches que requiert le maintien des personnes en perte d'autonomie dans leur milieu naturel?

La réflexion suivante livre une partie de la réponse: "Faire des visites et accompagner les personnes sont des actions qui nous rendent heureuses. Mais nous ne voulons pas exécuter des travaux domestiques ». Un même métier: pour soi, pour autrui! Tantôt chez soi, comme maîtresse de maison, tantôt à l'extérieur, comme aide-ménagère. Les bénévoles semblent contrariées par cette vocation qui semble la leur. D'ailleurs, selon une étude menée par Rita Therrien, il appert que, dans la société et dans la famille, ce sont les femmes qui ont la tâche de s'occuper des autres, d'accomplir des fonctions de prévention, de soin et de soutien en phase aiguë ou chronique, de santé communautaire 
et de services sociaux de groupe (1987:91). Toujours est-il que plusieurs bénévoles s'efforcent de découvrir, dans le bénévolat, la solution d'une difficulté inhérente à leur situation quotidienne: I'isolement. D'autres cherchent à se tourner vers le monde du travail. "Nos actions permettent à l'État de gérer à bon compte des problèmes sociaux considérables, mais nous souhaitons choisir nos activités. »Ce n'est pas d'aujourd'hui que les femmes font du bénévolat, mais de nouvelles motivations les animent et elles deviennent plus sélectives.

Il faut considérer un autre facteur pour expliquer le malaise ressenti par certaines bénévoles dans l'exercice de leur pratique. Dans le but de répondre aux demandes d'aide qui leur sont adressées, les groupes trouvent, par leur participation à la mesure Expérience de travail, une main-d'œuvre essentielle sans laquelle tout indique que le capital humain ferait dramatiquement défaut. Celle-ci, faut-il le rappeler, est une mesure de développement de l'employabilité offerte aux prestataires de la sécurité du revenu. Elle vise à favoriser chez les participants l'acquisition d'attitudes et d'habitudes de travail, afin de maintenir ou de développer leur employabilité tout en permettant I'acquisition de notions et d'expériences utiles sur le marché du travail (MSSS, 1992: 2). Le groupe bénévole qui les encadre reçoit cent dollars par mois pour chacun des participants. Les sommes recueillies permettent, certes, l'encadrement de ces derniers, mais servent également à couvrir les dépenses relatives au budget de fonctionnement ${ }^{5}$. Devant la mesure Expérience de travail - souvent désignée par l'acronyme "programme Extra »-, les opinions ne manquent pas de s'exprimer, avec une plus ou moins grande efficacité et selon les intérêts que I'on poursuit. Observer les enjeux sociaux qui se dégagent de ce programme, sans vouloir faire l'apologie d'un monde meilleur, dépasserait le cadre de cet article.

Qu'il suffise de dire, pour le moment, ce qu'en pensent les bénévoles. Elles signalent d'abord que la participation à ce programme gouvernemental permet à leur groupe $\mathrm{d}^{\prime}$ offrir une plus grande variété de services ${ }^{6}$. Toutefois, cette aventure leur amène certains commentaires. "Les bénévoles sont payées, qu'elles fassent leur travail », disent certains bénéficiaires aux femmes qui les visitent. D'où vient cette idée de la part des personnes aidées que les bénévoles sont rémunérées? La présence des participants à la mesure Expérience de travail n'est

5. Les organismes sans but lucratif recourent à la mesure Expérience de travail dans une proportion de $75 \%$. Les groupes rencontrés au cours de cette étude accueillent de 3 à 28 participants. 
pas étrangère à de telles réflexions. Il y aura lieu de s'interroger sur les conséquences qu'une telle perception peut engendrer à plus ou moins long terme dans l'univers du bénévolat qui se trouve menacé par l'impasse.

\section{Les exigences des bénéficiaires et la vente de services}

Le personnage que Romain Gary a créé autour du thème du bénévolat donne une image sinon d'ingratitude, au moins d'une exigence très poussée sur les attentes des personnes aidées. Il écrit en effet: «Je n'ai pas vu mademoiselle Cora pendant des jours. Elle m'avait appelé trois fois à S.O.S., mais je voulais passer le temps, pour ne pas trop I'habituer» (1979: 63). Loin d'accréditer l'évocation littéraire comme instrument de démonstration, il n'en demeure pas moins que s'y trouvent traduits des modes d'appréhension et de relations sociales qui s'apparentent au présent propos et peuvent servir d'illustration pour apporter une compréhension de ce que ce propos veut démontrer. En effet, plusieurs des bénévoles rencontrées (dix sur treize) réagissent à l'attitude intransigeante des bénéficiaires qui les chargent d'obligations de toutes sortes. Le caractère impératif qui accompagne les demandes les laisse pensives. Les femmes souhaitent que les bénéficiaires diminuent leurs attentes à l'égard d'autrui, et qu'à tout le moins ils se rappellent que certaines exigences s'accommodent mal d'un acte gratuit. Quelque étrange que soit cette réflexion sur les attentes des bénéficiaires, il est bon de dire ici qu'elle plonge ses racines dans la complexité des relations entre l'individu et ses semblables. En faire grief, c'est oublier de comprendre également que le contexte dans lequel s'exerce l'action bénévole s'apparente de plus en plus au travail professionnel.

Notamment, il est important de signaler que l'augmentation du nombre de demandes d'assistance conduit certains groupes bénévoles à adopter une conduite jusqu'alors impensable: la vente de leurs services (entretien ménager, deux dollars I'heure, transport, entre cinq et dix dollars). La plus grande partie des sommes recueillies lors des actions bénévoles - n'est-ce pas paradoxal de parler d'un geste gratuit rémunéré ! - sert à payer le nombre de kilomètres que les bénévoles parcourent avec leur voiture, dans l'exercice de leurs fonctions. Mais cette pratique semble embrouiller les bénéficiaires et les incite

6. Le soutien à domicile représente $29,5 \%$ des postes de travail de la mesure Expérience de travail et les personnes âgées bénéficient de plus de $57 \%$ des services (MSSS, 1992 : 9). 
à formuler d'autres revendications. En outre, les citoyens seront à même de se sentir d'autant plus appuyés que la nouvelle Loi sur les services de santé et les services sociaux explicite la légitimité de leurs revendications virtuelles en leur donnant un droit de recours. En effet, la loi 120 reconnaît que la personne utilisatrice de services en provenance du secteur communautaire pourra «formuler une plainte auprès de la Régie régionale sur les services qu'elle a reçus ou qu'elle aurait dû recevoir ». Ainsi, l'article 72 de cette loi sanctionne les groupes bénévoles dans leur rôle de services et, qui plus est, accorde au citoyen un droit de recours. Le bénévolat se doit donc d'être bien géré. Certes, les personnes qui composent les groupes de bénévoles s'unissent en vue d'un but déterminé: apporter un secours à autrui, le but plus lointain étant de maximiser cette aide. Cependant, n'y a-t-il pas quelque chose d'excessif dans cette recherche d'une gestion efficace du bénévolat?

\section{L'impact de la pratique bénévole sur l'emploi}

Certaines femmes interrogées (six sur treize) vont jusqu'à se demander si c'est une bonne idée de compter sur le bénévolat, puisque celui-ci peut constituer un danger pour l'emploi. Elles s'interrogent sur la pertinence de l'action bénévole, alors que le taux de chômage ne fait que s'élever. À ce propos, plusieurs syndiqués leur ont, tour à tour, signifié que cette pratique sociale constitue une menace à la création d'emplois. D'autres bénévoles (quatre sur treize) partagent cette réflexion et se disent mal à l'aise de discuter avec les travailleurs de certains établissements publics. À titre d'anecdote, la responsable du bénévolat dans un centre hospitalier rapporte qu'il lui a été impossible jusqu'à maintenant d'implanter un service de bénévoles à la salle d'urgence en raison du refus des employés syndiqués. Cette question n'a pas fini d'alimenter les débats, puisqu'il soulève des échanges d'arguments, des points contradictoires dans un contexte où règne la rareté de l'emploi. Alors que les bénévoles s'interrogent, les syndiqués, pour leur part, résistent. À preuve, le soutien financier accordé aux bénévoles dans l'exercice de leur pratique est dénoncé par les conducteurs de taxi qui exigent qu'un montant maximum soit décrété par la Loi modifiant la Loi sur le transport par taxi (loi C-62, 1993). Les données recueillies ont mis au jour plusieurs contraintes qui affectent l'exercice bénévole. II s'agit, dans cette foulée, d'exposer le point de vue des femmes sur la façon dont elles relèvent le défi de leurs responsabilités organisationnelles. 


\section{LA SÉDUCTION DU LIEN SOCIAL}

"Tant qu'on aime on est utile, tant qu'on se sent aimé on est indispensable », voilà d'entrée de jeu ce qu'une bénévole exprime pour expliquer sa participation dans un groupe. Il est intéressant de noter également que la majorité des personnes rencontrées affirment qu'en aidant les autres on s'aide soi-même. Certaines l'expriment ouvertement : " Nous recevons des leçons d'amour et d'amitié. » Cependant, même si certaines pressions sont parfois exercées sur les bénévoles afin de les inciter à s'orienter vers un type donné de bénéficiaires, il n'en demeure pas moins qu'elles jouissent, la plupart du temps, d'une latitude dans le choix de leurs activités. En effet, dix des treize bénévoles rencontrées trouvent que leur marge de manœuvre est assez large, ce qui signifie qu'elles ont la possibilité de faire ce qui leur plaît au moment jugé opportun. Il devient difficile de vendre l'idée de visiter des personnes âgées dans un centre d'accueil à des femmes qui souhaitent travailler dans un comptoir vestimentaire. "Je ne me sens pas contrôlée et cela est essentiel pour moi », est une remarque fréquente (neuf sur treize). Ainsi, au-delà de ce commentaire, il faut souligner toute l'importance que les bénévoles accordent à leur liberté d'action. Une telle observation renvoie au constat des dirigeantes sur la nécessité de choisir ses actions, comme source importante de stimulation pour les personnes qui veulent faire du bénévolat. À ce propos, les facteurs de motivation les plus exprimés sont les suivants :

- me sentir utile (6);

- apporter un peu de bonheur aux autres (3);

- faire des actions constructives qui apportent une valorisation (2);

- rendre ce que j'ai reçu; c'est un dû, une juste compensation ;

- occuper mon temps libre (1).

Les bénévoles avouent avoir besoin d'un climat amical et bienveillant pour se sentir à l'aise. Une atmosphère de confiance et de cordialité favorise les communications entre les différentes personnes engagées dans l'action. À ce titre, les dirigeantes jouent un rôle crucial dans le développement de la cohésion du groupe dans un premier temps, et dans son évolution par la suite. Les bénévoles expriment des attentes à l'égard des dirigeantes, les espérant attentives, positives, franches et vigilantes. Enfin, quelques femmes (trois sur treize) souhaitent que les responsables puissent montrer que le bénévolat participe à l'histoire, à l'actualité et au devenir de la communauté. 
Les opérations liées à l'accomplissement de la pratique bénévole comme conditions de sa validité commandent de nouvelles obligations. Quels sont donc les facteurs qui pourraient expliquer, du moins en partie, le désir d'une pratique bénévole à l'intérieur de ces groupes? Selon les commentaires recueillis, il faut chercher la source dans la profondeur des liens sociaux. D'ailleurs, le partage de sentiments comme ciment de la vie sociale a été soulevé par maints auteurs. II est constitutif de toute la sociologie elle-même des auteurs qui, d'Émile Durkheim à Georges Gurvitch, pour ne citer que ceux-là, ont montré que la société $n^{\prime}$ est rien d'autre qu'une fabrique de relations s'exprimant dans une plénitude infinie de manifestations dont le bénévolat est peutêtre finalement la base. Car, pour être ensemble, il faut nécessairement se fonder sur une logique de partage et d'échanges, jamais réductibles à ce qui est exclusivement monnayable. Le sentiment $d^{\prime}$ appartenance au groupe constitue donc, pour plusieurs, une source de stimulation importante dans leur engagement. Certaines vont jusqu'à préciser que celui-ci sert de balise aux abus qui les guettent parfois en leur donnant, par exemple, le droit de refuser une activité.

Devant cet élément d'encadrement souhaité, il faut être attentif pour jauger la pertinence de relations harmonieuses entre les dirigeantes et les bénévoles. Les témoignages de respect, de reconnaissance et l'hommage rendu aux bénévoles au cours d'activités spéciales constituent pour plusieurs une source d'encouragement, même si, à l'occasion, certaines (trois sur treize) se disent gênées par de telles manifestations. En effet, pour ces dernières, l'aide apportée constitue la gratification première et $c^{\prime}$ est la seule jugée acceptable pour une plus grande congruence envers soi-même. "Le bénévolat comporte sa propre récompense », explique une bénévole. Mais, en tout cas, il s'agit là d'une valorisation dont on n'a pas fini de souligner l'importance dans l'aventure bénévole.

«Faire du bénévolat, c'est donner un petit peu plus que les professionnels ne donnent, comme l'organisation des fêtes de Noël, l'accompagnement à l'église, la récupération de vêtements et bien d'autres choses encore", livrent les bénévoles. Kouchner écrit: "Le bénévolat autorise le rêve que le travail salarié étouffe» (1986:228). Du reste, l'étonnante vitalité dont font preuve plusieurs femmes dans I'exercice des différentes activités peut même surprendre. Mais n'estelle pas l'indice d'un dynamisme renouvelé dans tous les domaines de la vie sociale? Néanmoins, les questions surgissent en quelque sorte à l'intérieur de chacune des bénévoles dans la mesure où le désir d'apporter une aide à autrui se voit aussi débordé par les exigences du geste. Cet ajustement dans la manière d'exercer cette pratique d'attention 
à l'autre, dès lors que les groupes deviennent plus soucieux de leur fonctionnalité, incite-t-il les bénévoles à persévérer dans leurs activités?

Le paradoxe de toute institution est d'encadrer le rendement par la multiplication des contraintes. II n'y a peut-être pas, dans cette assertion, de loi susceptible d'application universelle, mais au moins I'instrument d'une conjecture. L'interrogation qui l'interpelle demande toutefois une validation, un amendement s'il y a lieu, de la réflexion par l'interprétation des phénomènes. Si instituer est reconnaître des formes sociales et contraindre, obliger à des règles de fonctionnement, ne peut-on pas dire que l'aspiration à maximiser le rendement comporte une menace virtuelle de contre-productivité, dès lors que la lourdeur des mécanismes mis en place risque d'avoir des effets dissuasifs? La lecture des chiffres invite ici à soutenir le contraire, puisque les treize bénévoles interrogées ont renouvelé leur intention de continuer à œuvrer au sein du groupe, malgré les contraintes posées par les institutions. Mais proclamer l'allégeance ne se fait pas sans protester: critique des formalités prescrites, désapprobation des changements survenus, nostalgie d'une spontanéité perdue, etc., ne sont que quelques indicateurs d'une crainte, au demeurant contrôlée, puisqu'elle $\mathrm{n}^{\prime}$ inhibe pas, apparemment, les engagements dans le groupe, du moins pour l'instant.

Ce qui peut paraître irrationnel en première analyse ne l'est pas à y regarder de près. $\mathrm{D}^{\prime}$ abord, les bénévoles ont un intérêt à maintenir leur engagement: elles en retirent des bénéfices, comme celui d'avoir un réseau de relations étendu. L'attachement au milieu, I'innovation et le dynamisme constituent aussi un ensemble de facteurs qui militent en faveur de leur participation. Ensuite, elles réussissent assez bien à contourner les nouvelles exigences: I'ajustement des groupes bénévoles se situe plus sur le plan de la structure que sur le plan de la pratique elle-même. Donc, les bénévoles, dans leur quotidien, réussissent à se soustraire aux changements, parfois à s'y adapter, en nouant les impératifs du cœur aux contraintes de la raison. Toujours est-il que, vu de l'intérieur, le travail demeure suffisamment intéressant pour que les bénévoles se disent prêtes à poursuivre leur engagement. Néanmoins, la diminution du nombre de bénévoles, les difficultés de recrutement, le recours aux programmes d'employabilité afin de combler un manque de ressources humaines, tous ces facteurs appellent une certaine nuance dans cette conclusion.

Mais "l'État peut-il tuer le bénévolat?», comme l'exprime une bénévole inquiète pour l'avenir de sa pratique. Les limites de la connaissance théorique se doublent ici d'une mise en garde sur les fausses limpidités que suggèrent parfois penseurs et praticiens, militants et bénévoles, membres et permanents des structures, au sujet du rôle de 
I'État et des groupes. II faut alors comprendre que le présent propos s'inscrit dans l'analyse des transformations des réseaux bénévoles, œuvrant dans le domaine de la santé et des services sociaux. Ainsi, le découpage sélectif de l'objet d'étude fait perdre en extension conceptuelle ce que l'on gagne en compréhension. Or, le bénévolat se déploie dans des sphères diverses qui n'éliminent pas ses caractéristiques propres, comme sa définition l'inspire. L'étude peut s'avérer un apport pour saisir les conséquences de l'institutionnalisation des réseaux. Mais la réalité du bénévolat $n^{\prime}$ est pas réductible à la logique d'un système. Des réseaux complexes, des relations informelles, des fils anonymes tissent également la trame de cette pratique sociale. Tenir compte de ce discours plus que nous l'avons fait ici serait sans doute une avenue à explorer.

\section{Références bibliographiques}

BLANCHET, J. (1990). La gestion du bénévolat. France: Economica; Canada: Agence $d^{\prime}$ Arc.

FERRAND-BECHMANN, D. (1992). Solidarité et bénévolat. Paris : Éditions SyrosAlternative.

CARPENTIER, J. et F. VAILLANCOURT ( 1990). L'activité bénévolat au Québec. La situation en 1987 et son évolution depuis 1979. Québec: Les Publications du Québec.

DuCHESNE, D. (1989). Donner sans compter: les bénévoles au Canada. Canada, Division de l'analyse des enquêtes sur le travail et les ménages.

GARY, R. (1979). L'angoisse du roi Salomon. Paris: Mercure de France.

GOUVERNEMENT DU QUÉBEC (1991). Loi sur les services de santé et les services sociaux et modifiant diverses dispositions législatives. Québec : Éditeur officiel du Québec.

Gouvernement du QuÉBEC (1992). Expérience de travail. Dans le réseau de la santé et des services sociaux. Rapport d'évaluation. Ministère de la Santé et des Services sociaux.

KOUCHNER, B. (1986). Charité Business. France : Éditions Belfond, Pré-auxClercs.

LAFOREST, M. et B. REDJEB (1989). L'intervention sociale non salariée. Étude exploratoire de diverses pratiques d'assistance bénévole auprès des personnes âgées dans la région de Québec. Sainte-Foy, Université Laval.

LAMOUREUX, H. (1991). L'intervention sociale collective. Une éthique de solidarité. Québec: Éditions du Pommier.

LAROCHELlE, G. et S. ROBICHAUD (1991). "Contestation et attestation étatique: le nouveau catéchisme des groupes communautaires du Québec », Revue française de science politique, Presses de la Fondation nationale des sciences politiques, vol. 41, no $5: 649-674$. 
PAyetTe, M. et F. VAiLlancourt (1983). Le portrait des bénévoles québécois. Centre de recherche en développement économique, Université de Montréal.

RoBiChaUd, S. (1994). L'État et les solidarités bénévoles, les enjeux politiques de la gratuité. Thèse présentée à l'École des gradués de I'Université Laval, Faculté des sciences sociales, Département de science politique.

THERRIEN, R. (1987). La contribution informelle des femmes aux services de santé et services sociaux. Rapport de recherche et synthèse critique présentés à la Commission Rochon, $\mathrm{n}^{\circ} 8$. 